\title{
The relation between sarcomere energetics and the rate of isometric tension relaxation in healthy and diseased cardiac muscle
}

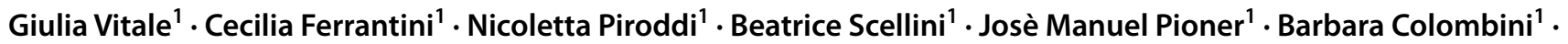 \\ Chiara Tesi ${ }^{1} \cdot$ Corrado Poggesi $^{1}$ (D)
}

Received: 2 September 2019 / Accepted: 13 November 2019 / Published online: 19 November 2019

(C) The Author(s) 2019

\begin{abstract}
Full muscle relaxation happens when $\left[\mathrm{Ca}^{2+}\right]$ falls below the threshold for force activation. Several experimental models, from whole muscle organs and intact muscle down to skinned fibers, have been used to explore the cascade of kinetic events leading to mechanical relaxation. The use of single myofibrils together with fast solution switching techniques, has provided new information about the role of cross-bridge (CB) dissociation in the time course of isometric force decay. Myofibril's relaxation is biphasic starting with a slow seemingly linear phase, with a rate constant, slow $k_{\mathrm{REL}}$, followed by a fast monoexponential phase. Sarcomeres remain isometric during the slow force decay that reflects CB detachment under isometric conditions while the final fast relaxation phase begins with a sudden give of few sarcomeres and is then dominated by intersarcomere dynamics. Based on a simple two-state model of the CB cycle, myofibril slow $k_{\mathrm{REL}}$ represents the apparent forward rate with which CBs leave force generating states $\left(g_{\text {app }}\right)$ under isometric conditions and correlates with the energy cost of tension generation (ATPase/tension ratio); in short slow $k_{\mathrm{REL}} \sim g_{\mathrm{app}} \sim$ tension cost. The validation of this relationship is obtained by simultaneously measuring maximal isometric force and ATP consumption in skinned myocardial strips that provide an unambiguous determination of the relation between contractile and energetic properties of the sarcomere. Thus, combining kinetic experiments in isolated myofibrils and mechanical and energetic measurements in multicellular cardiac strips, we are able to provide direct evidence for a positive linear correlation between myofibril isometric relaxation kinetics (slow $k_{\mathrm{REL}}$ ) and the energy cost of force production both measured in preparations from the same cardiac sample. This correlation remains true among different types of muscles with different ATPase activities and also when CB kinetics are altered by cardiomyopathy-related mutations. Sarcomeric mutations associated to hypertrophic cardiomyopathy (HCM), a primary cardiac disorder caused by mutations in genes encoding sarcomeric proteins, have been often found to accelerate $\mathrm{CB}$ turnover rate and increase the energy cost of myocardial contraction. Here we review data showing that faster $\mathrm{CB}$ detachment results in a proportional increase in the energetic cost of tension generation in heart samples from both HCM patients and mouse models of the disease.
\end{abstract}

Keywords Muscle mechanics $\cdot$ Muscle energetics $\cdot$ Cardiac muscle $\cdot$ Myofilaments $\cdot$ Myosin $\cdot$ Troponin $\cdot$ Hypertrophic cardiomyopathy

\section{Introduction}

The mechanical performance of cardiac muscle results from the interplay between two macromolecular systems: (1) membrane bound-calcium handling proteins, responsible

Corrado Poggesi

corrado.poggesi@unifi.it

1 Department of Experimental and Clinical Medicine, University of Florence, Viale Morgagni 63, 50134 Florence, Italy for the $\mathrm{Ca}^{2+}$ signal that starts and stops contraction; (2) sarcomeric proteins, responsible for active and passive force generation and for contraction regulation by $\mathrm{Ca}^{2+}$. To dissect the relative roles of these two systems in the extent and kinetics of striated muscle contraction and relaxation, disparate preparations at all levels of the structural hierarchy have been used.

Though most models about the biochemical steps of crossbridge (CB) cycle and their regulation are based on studies of proteins in solution, isolated proteins lack the structural complexity and mechanical constraints of muscle 
fibers. Myofibrils, formed by serially arranged sarcomeres, maintain a complete structured ensemble of contractile and $\mathrm{Ca}^{2+}$ regulatory proteins. Thanks to the rapid equilibration with the medium, myofibrils allow the investigation of $\mathrm{CB}$ kinetic processes (for reviews see Poggesi et al. 2005; Stehle et al. 2009; Stehle and Tesi 2017). The possibility to easily obtain myofibrils from small human cardiac samples also provide a useful model to study disease-related mechanical and kinetic dysfunction in human cardiac sarcomeres (Neagoe et al. 2002; Piroddi et al. 2007, 2019; Belus et al. 2008, 2010; Walker et al. 2011; Witjas-Paalberends et al. 2014a; Vikhorev et al. 2017; Jeong et al. 2018).

Familial hypertrophic cardiomyopathy (HCM) is the most common inherited heart disease with a prevalence of 1:500 (Maron et al. 2014). It is considered a disease of the sarcomere because several genes encoding cardiac myofilament proteins have been shown to cause the disease (Watkins et al. 2011; Poggesi and Ho 2014). Some HCM-associated sarcomeric mutations primarily result in aberrant/faster CB dynamics and increased ATP usage for the force generation process (Belus et al. 2008; Witjas-Paalberends et al. 2014a; Ferrantini et al. 2017; Piroddi et al. 2019). It has been suggested long ago that sustained energy impairment in cardiomyocytes may be central in HCM disease (Crilley et al. 2003; Javadpour et al. 2003; Ashrafian et al. 2003).

Since the origin of the coupling between mechanic and energetic events during cardiac sarcomere contraction resides in the rates governing $\mathrm{CB}$ turn over, myofibril mechanic experiments associated with mechanic and energetic experiments in skinned muscle strips, represent unique tools to dissect the physiology and pathophysiology of sarcomere dynamics and energetics.

\section{Myofibrils and fast solution switching: experimental tools to investigate apparent CB kinetics}

While the complete cellular physiology of muscle contraction and relaxation can be only studied in intact muscle preparations, dissecting the roles played by CBs and myofilament regulation in the kinetics of the twitch is obscured by the relatively slow rates of myoplasmic $\mathrm{Ca}^{2+}$ rise and removal (Caputo et al. 1994; Gao et al. 1998; Bers 2001). To focus on the process of force generation and relaxation and its regulation at the sarcomere level, skinned muscle preparations are to be employed. Indeed, the removal of all plasmatic and Sarcoplasmic Reticulum membranes allows investigators to experimentally control the $\left[\mathrm{Ca}^{2+}\right]$ in the surrounding solution and focus on the process of force generation and relaxation at myofilament level. Caged $\mathrm{Ca}^{2+}$-compounds and caged $\mathrm{Ca}^{2+}$-chelators can be used in skinned cardiac muscle preparations to abruptly change the $\left[\mathrm{Ca}^{2+}\right]$ by flash photolysis and dissect the myofilament mechanisms of force activation and force relaxation from those related to myoplasmic $\mathrm{Ca}^{2+}$ handling (e.g. Palmer and Kentish 1998). The use of caged compounds, however, is not free from problems. Caged compounds have general inherent limitations with respect to the input of large radiant energies and the production of reactive photolytic by-products as well as uncertainties in the actual concentration of the compounds inside the skinned muscle preparations. Moreover, the kinetics of force activation and relaxation require different caged compounds and must be studied separately (see Palmer and Kentish 1998). If we want to focus on the relaxation process one significant additional limit of caged compounds is that the increased $\mathrm{Ca}^{2+}$ buffering capacity following photolysis of caged $\mathrm{Ca}^{2+}$-chelators is not enough to induce a complete relaxation from high levels of activation (Wahr et al. 1998; Luo et al. 2002).

Isolated myofibrils, as a model for mechanical experiments, hold some advantages over larger preparations and are ideal to obtain insight about the CB kinetics during an activation-relaxation cycle (e.g. Poggesi et al. 2005). Isolated myofibrils are the smallest subdivision of the contractile apparatus of striated muscle that retain the organized filament lattice and entire ensemble of associated proteins. Because of their small size (the diffusion distances are $<2 \mu \mathrm{m})$, rapid $(<1 \mathrm{~ms})$ equilibration is ensured with surrounding solution and thus, the myofilament lattice can be effectively clamped at any desired composition by flowing streams of solution over the preparation. Rapid solution switching methods in myofibrils have given straightforward insights into the kinetics of force activation and relaxation following abrupt increase and decrease of $\left[\mathrm{Ca}^{2+}\right]$ (for a review see Stehle et al. 2009).

The abrupt rise of $\left[\mathrm{Ca}^{2+}\right]$ by fast solution switching in human and mouse ventricular myofibrils at optimum overlap (see Fig. 1A, B) initiates tension development processes that are qualitatively similar but with much different rates. At maximal $\mathrm{Ca}^{2+}$ activation, tension rises approximately mono-exponentially, with a rate constant $k_{\mathrm{ACT}}$ that is one order of magnitude faster in the mouse ventricular myofibril compared to the human preparation (Fig. 1C). Under steadystate conditions of force generation a large mechanical perturbation is applied to the myofibrils aimed at detaching most of the attached force generating CBs (Fig. 1A, B). This mechanical perturbation drops force to zero before starting a force redevelopment process, with rate $k_{\mathrm{TR}}$, that is thought to reflect the apparent $\mathrm{CB}$ turnover rate under conditions of steady-state activation (Brenner 1988). Under our usual experimental conditions, $k_{\mathrm{ACT}}$ is the same as $k_{\mathrm{TR}}$, indicating that thin filament $\mathrm{Ca}^{2+}$-activation is a rapid process and that both kinetic parameters reflect the apparent rate of $\mathrm{CB}$ turn-over.

Full force relaxation of human and mouse ventricular myofibrils, induced by rapid reduction in $\left[\mathrm{Ca}^{2+}\right]$ below contractile threshold, is markedly biphasic (Fig. 1D), like in all myofibril types studied so far. The behaviour parallels that 
A

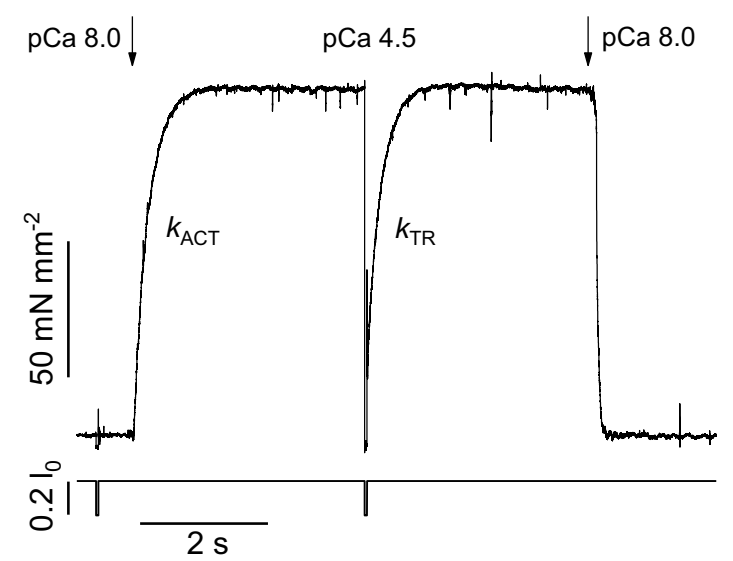

C pCa 8.0 , pCa 4.5

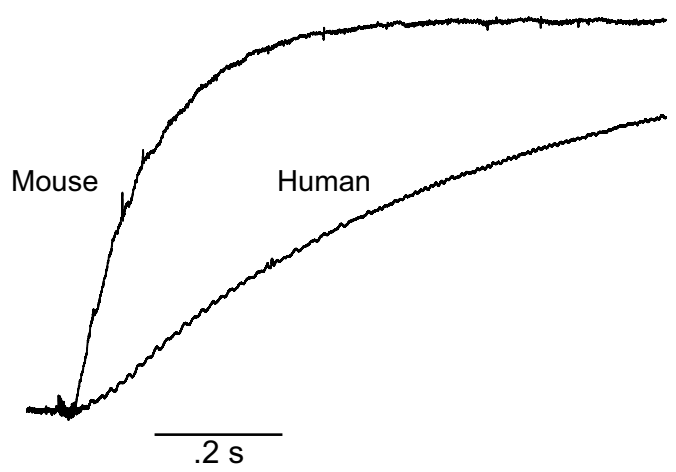

Fig. 1 Tension activation and relaxation of mouse and human ventricular myofibrils. A, B Top traces, representative tension records of mouse (A) and human (B) ventricular myofibrils maximally $\mathrm{Ca}^{2+}$-activated and fully relaxed by fast solution-switching $\left(15^{\circ} \mathrm{C}\right)$. Resting sarcomere length $2.2 \mu \mathrm{m}$. The arrows mark the start of the solution changes that modify the myofibril $\mathrm{pCa}$ as indicated. Bottom traces are length signals showing the release-restretch protocol applied to the myofibrils under conditions of steady $\mathrm{Ca}^{2+}$-activation. $k_{\mathrm{ACT}}$ is the rate constant of tension development following maximal $\mathrm{Ca}^{2+}$ activation; $k_{\mathrm{TR}}$ is the rate constant of tension redevelopment following release-restreach protocol during steady-state $\mathrm{Ca}^{2+}$ activation.

C, D Same traces as in A, B superimposed on a faster time base after

previously described in intact skeletal muscle fibres relaxing from maximum tetanic force (Huxley and Simmons 1970, 1973; Cleworth and Edman 1972; Edman and Flitney 1982). Force relaxation starts with a slow, seemingly linear, phase with a rate constant slow $k_{\mathrm{REL}}$ that is thought to reflect the apparent rate of $\mathrm{CB}$ detachment under isometric conditions also because in myofibril experiments the bulk of the slow relaxation phase occurs after $\left[\mathrm{Ca}^{2+}\right]$ has been effectively reduced below contractile threshold (Poggesi et al. 2005). The slow force decay is followed by a rapid and exponential decay of force, with a rate constant fast $k_{\mathrm{REL}}$. While myofibril sarcomeres remain isometric during the early slow relaxation phase, the final fast force decay is initiated when a few, mechanically weaker, sarcomeres rapidly lengthen
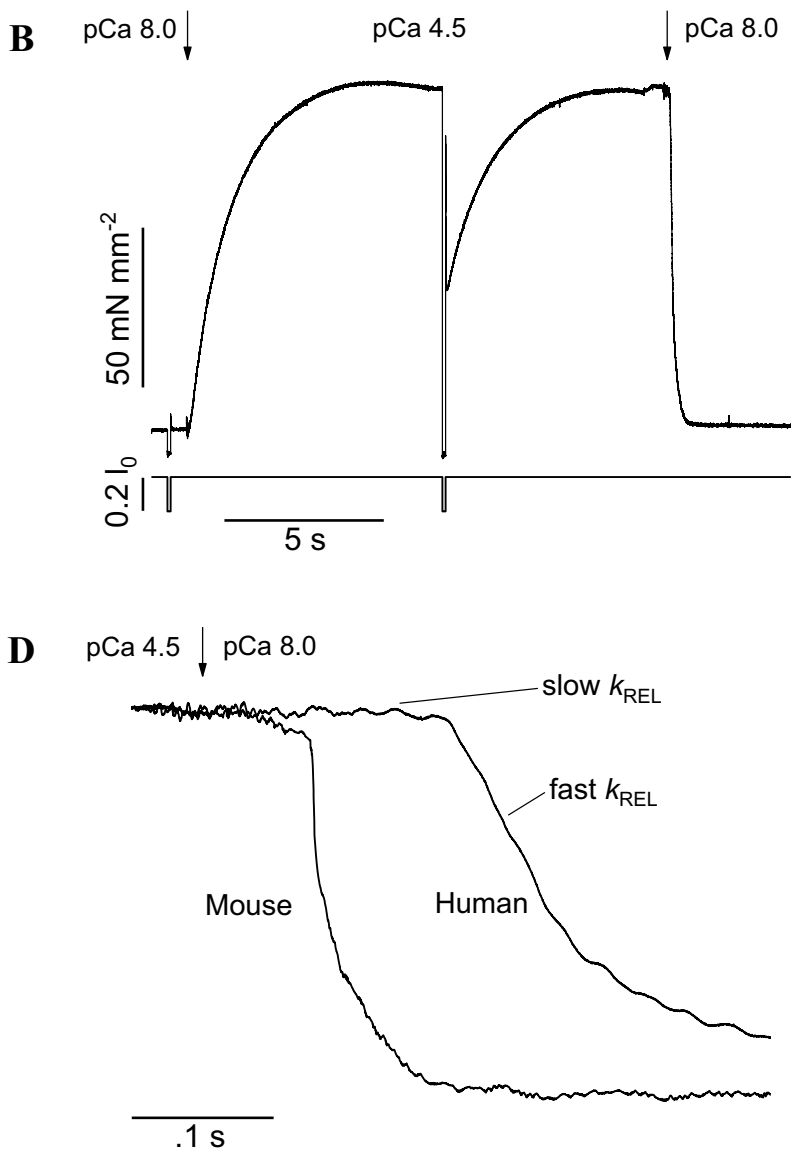

normalization for maximal tension to highlight force activation $(\mathbf{C})$ and relaxation (D) kinetics. As shown in $\mathbf{D}$ full tension relaxation from maximal activation is biphasic both in mouse and human myofibrils. The rate constant of the early slow force decline (slow $k_{\mathrm{REL}}$ ) is estimated from the slope of the regression line fitted to the force trace normalized to the entire amplitude of the force relaxation transient. The rate constant for the final fast phase of tension decline (fast $k_{\mathrm{REL}}$ ) is estimated from mono-exponential fit. Of note, both the kinetics of force activation $(\mathbf{C})$ and force relaxation (D) are much faster in the mouse compared to the human ventricular myofibril indicating faster cross-bridge turnover and faster detachment rate respectively

(sarcomere "give") and is then dominated by inter-sarcomere dynamics (Stehle et al. 2002; Poggesi et al. 2005). Overall force relaxation is much faster in the mouse compared to the human preparation (Fig. 1D).

Previous studies in myofibrils have shown that CBs are the major determinants of the kinetics of tension activation and of the early isometric phase of relaxation whereas the regulatory $\mathrm{Ca}^{2+}$ switch is a much faster process (Poggesi et al. 2005; Stehle et al. 2009). Some of the evidence collected during the last years in favour of the dominant role of $\mathrm{CBs}$ in myofibril tension kinetics are listed below.

(i) Under the conditions of our myofibril experiments (maximal $\mathrm{Ca}^{2+}$-activation, sarcomere length $2.20 \mu \mathrm{m}$, 
low $\left[\mathrm{P}_{\mathrm{i}}\right]$, relatively low temperature $\left.5-15^{\circ} \mathrm{C}\right) k_{\mathrm{ACT}}$ and $k_{\mathrm{TR}}$ are essentially identical in a variety of preparations that largely differ in tension kinetics (Fig. 2). As already mentioned above, $k_{\mathrm{TR}}$ is measured under quasi-steady state conditions of thin filament activation and is thought to reflect $\mathrm{CB}$ turnover; $k_{\mathrm{ACT}}$ could in principle be a slower process as it may also be limited by thin filament activation kinetics. As shown in Fig. 2, $k_{\mathrm{ACT}}$ never falls below the identity line with $k_{\mathrm{TR}}$ strongly suggesting that both parameters primarily reflect $\mathrm{CB}$ turnover rate whereas $\mathrm{Ca}^{2+}$-activation is a much faster process.

(ii) The lack of a significant influence of thin filament activation/inactivation kinetics on myofibril tension activation and relaxation kinetics was also clear from the comparison between fluorescence stopped flow studies on cardiac myofibril suspensions and mechanical studies on single cardiac myofibrils. The results (Solzin et al. 2007 reviewed by Stehle et al. 2009) showed that the structural changes in cardiac Troponin (Tn) following calcium binding to or dissociation from cardiac $\mathrm{TnC}$ in response to sudden

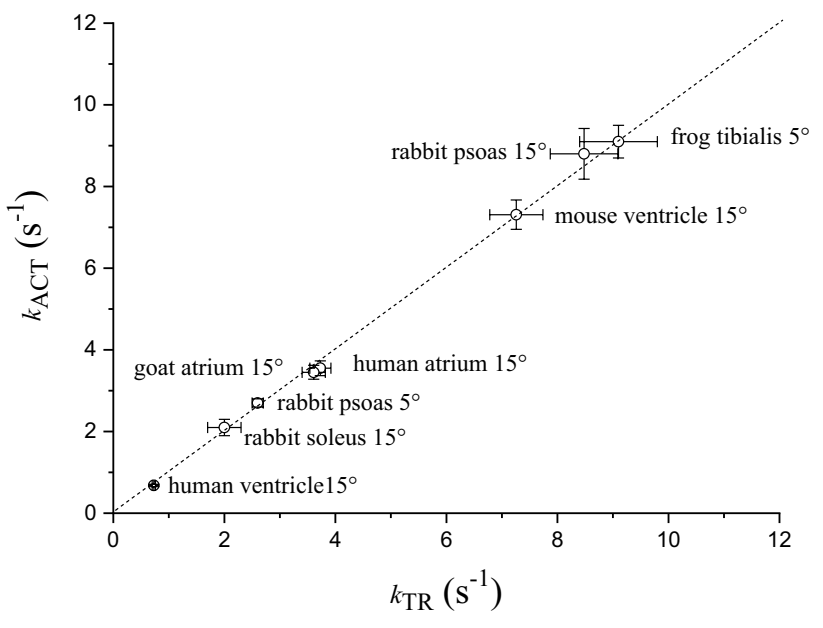

Fig. 2 Rates of tension activation and tension redevelopment in different types of maximally $\mathrm{Ca}^{2+}$-activated skeletal and cardiac myofibril preparations at 5 or $15{ }^{\circ} \mathrm{C}$. Mean \pm SEM $(\mathrm{n} \geq 11)$. Dotted line, identity line. Unpublished data increase or decrease in calcium (Tn switch) are much faster than myofibril force activation and relaxation kinetics.

(iii) Tn replacement studies in myofibrils further support the idea that contraction-relaxation myofibril kinetics, following abrupt increase/decrease in $\left[\mathrm{Ca}^{2+}\right]$, are mainly set by the intrinsic $\mathrm{CB}$ cycling rate and are not directly altered by the kinetics of the Tn switch (Piroddi et al. 2003; de Tombe and Stienen 2007). Notably, as shown in Fig. 1 and Table 1, the kinetics of force activation and relaxation in mouse ventricular myofibrils are almost tenfold faster than in human ventricular myofibrils. However, exchange of human cardiac $\mathrm{Tn}$ for the endogenous mouse cardiac $\mathrm{Tn}$ in mouse ventricular myofibrils does not slow down force kinetics (see Table 1) indicating that it is the myosin motor form that sets the force kinetics of cardiac sarcomeres. Studies of replacement of all thin filament regulatory proteins ( $\mathrm{Tn}$ and Tropomyosin) in myofibrils confirm the idea of a dominant role of CB cycling rate in determining the kinetics of sarcomere tension activation and relaxation (Siththanandan et al. 2009; Scellini et al. 2010).

(iv) Changes in the concentrations of substrate and products of the acto-myosin ATPase markedly affect myofibril force activation and relaxation kinetics (Tesi et al. 2002). For instance, inorganic phosphate accelerates force kinetics in cardiac myofibrils; in particular it increases the rate of the slow force decay, consistent with the idea that slow $k_{\mathrm{REL}}$ reflects the dissociation of force generating CBs to nonforce-generating states via both forward and backward transitions (Stehle and Tesi 2017). At variance with the large impact of interventions that interfere with key CB transitions, interventions that increase myofilament $\mathrm{Ca}^{2+}$-sensitivity by altering the $\mathrm{Ca}^{2+}$ Tn switch usually do not significantly affect $k_{\mathrm{ACT}}$, $k_{\mathrm{TR}}$, and slow $k_{\mathrm{REL}}$ measured in cardiac myofibrils at maximal activation (Piroddi et al. 2007; Kreutziger et al. 2011).

Table 1 Kinetic parameters of mouse and human ventricular myofibrils following replacement by exchange of the endogenous cTn complex with human recombinant $\mathrm{cTn}$

\begin{tabular}{llccc}
\hline Myofibril type & $k_{\mathrm{ACT}}\left(\mathrm{s}^{-1}\right)$ & $k_{\mathrm{TR}}\left(\mathrm{s}^{-1}\right)$ & Slow $k_{\mathrm{REL}}\left(\mathrm{s}^{-1}\right)$ & Fast $_{\mathrm{REL}_{2}}\left(\mathrm{~s}^{-1}\right)$ \\
\hline Mouse Ventr (control) & $7.35 \pm 0.54$ & $7.12 \pm 0.61$ & $1.45 \pm 0.21$ & $28.3 \pm 2.9$ \\
Mouse Ventr (human cTn exchanged) & $7.05 \pm 0.44$ & $6.92 \pm 0.75$ & $1.55 \pm 0.28$ & $25.3 \pm 3.1$ \\
Human Ventr (human cTn exchanged) & $0.84 \pm 0.03$ & $0.78 \pm 0.04$ & $0.30 \pm 0.02$ & $4.65 \pm 0.19$ \\
\hline
\end{tabular}

Data are mean $\pm \mathrm{SEM} ; \mathrm{n} \geq 10$. Data from human $\mathrm{cTn}$ exchanged mouse myofibrils and control (sham-treated) mouse myofibrils are unpublished data; data from human cTn exchanged human ventricular myofibrils are from Piroddi et al. (2019). The exchange protocol allows replacement of $70-90 \%$ of the endogenous cTn complex 
To better interpret myofibril tension kinetic parameters in terms of apparent CB kinetics we need a description of the reaction pathway for acto-myosin ATPase and energy-transduction cycle. The reaction pathway is usually described as a series of coupled biochemical and mechanical events with a relatively large number of transitions (Gordon et al. 2000; see also Fig. 5 in Ferrantini et al. 2009). Generally accepted schemes can be reduced for our purposes to a simpler twostate CB scheme (Brenner 1988). On the basis of myosin's binding affinity for actin, two general types of CBs can be defined in the two-state model of acto-myosin interactions: $\mathrm{AM}_{\text {no force }}$ that represents all detached and "weak binding states" (detached states M.ATP, M.ATP.Pi in rapid equilibrium with AM.ATP, AM.ADP.Pi) and $\mathrm{AM}_{\text {force }}$ that represents all "strong binding states" (AM, AM*.ADP). The exoergonic release of inorganic phosphate $\left(\mathrm{P}_{\mathrm{i}}\right)$ is thought to power the working stroke. The transition from the nonforce-generating states to the force generating states has an overall apparent rate constant $f_{a p p}$. It is well known that at physiological $\left[\mathrm{P}_{\mathrm{i}}\right]$ the power stroke reverse transition may occur so that the return to the non force generating states may occur both through the backward transition $\left(\mathrm{P}_{\mathrm{i}}\right.$ rebinding and reversal of the power stroke) and the forward transition (ADP release and ATP binding). The apparent rate constant for the forward transition is $g_{\text {app }}$ while the apparent rate constant for the reverse transition of the force generating step is $f_{a p p}^{\prime}$ that depends on [Pi].

$\mathrm{AM}_{\text {No Force }} \underset{f_{a p p}^{\prime}}{\stackrel{f_{a p p}}{\rightleftarrows}} \mathrm{AM}_{\text {Force }} \stackrel{g_{a p p}}{\rightarrow} \mathrm{AM}_{\text {No Force }}$

In the virtual absence of $\mathrm{P}_{\mathrm{i}}$, that is the experimental condition of most myofibril studies, the apparent rate constant for the reverse transition $f_{\text {app }}^{\prime}$ can be neglected. Thus, during myofibril relaxation, following $\mathrm{Ca}^{2+}$ removal, strongly bound $\mathrm{CBs}$ can leave the force-generating states only via forward transitions whereas weakly-bound $\mathrm{CBs}$ are unable to go into the force-generating states (Poggesi et al. 2005; Ferrantini et al. 2009). In terms of CB turnover kinetics, myofibril slow $k_{\mathrm{REL}}$ measured in the absence of $\mathrm{P}_{\mathrm{i}}$ consists solely of forward CB detachment, that leads to ATP hydrolysis steps, continuing at the same rate as during the isometric contraction; in short slow $k_{\mathrm{REL} \sim} g_{\text {app }}$.

According to this model, the overall CB turnover rate, given by $f_{\text {app }}+g_{\text {app }}$, is reflected by $k_{\mathrm{ACT}}$ and $k_{\mathrm{TR}}$, maximal tension is proportional to $f_{\text {app }} /\left(f_{\text {app }}+g_{\text {app }}\right)$, ATPase activity is proportional to $f_{\text {app }} \cdot g_{\text {app }} /\left(f_{\text {app }}+g_{\text {app }}\right)$, and the energy cost of tension generation (ATPase/tension ratio) is proportional to $g_{\text {app }}$ (Brenner, 1988; de Tombe and Stienen 2007). Given slow $k_{\mathrm{REL}} \approx g_{\text {app }}$, an increase/decrease in slow $k_{\mathrm{REL}}$ should be able to predict an increase/decrease in the amount of ATP spent to generate a given amount of isometric force (i.e. the energy cost of tension generation).
Direct and coupled measurements of isometric tension and ATPase activity under isometric conditions can unequivocally confirm this conclusion.

\section{Cardiac sarcomere energetics and the correlation between energy cost of tension generation and the isometric relaxation rate of cardiac myofibrils from different preparations}

Chemically permeabilized cardiac muscle strips, isolated from human and animal hearts, are the conventional preparations used to measure the isometric rate of ATP consumption of the sarcomeric proteins and to directly correlate the amount of ATP hydrolyzed to the amount of developed force (i.e. the tension cost of cardiac contraction) (de Tombe and Stienen 1995, 2007; Narolska et al. 2005; Witjas-Paalberends et al. 2014a, b). The removal of virtually all membrane structures leaves the sarcomeric proteins (i.e. myosin motors) as the only source of energy consumption in these preparations and this allows us to circumvent many of the problems that hamper myothermal measurements on intact myocardium (Holubarsch et al. 1991; Alpert et al. 1991; de Tombe and Stienen 2007). Indeed, interpretation of myothermal experiments in intact preparations can be mainly complicated by uncertainties in the relative amount of total energy that is used by the excitation contraction coupling system or by the sarcomeric proteins (Gibbs et al. 1988). It is to be noted, however, that in recent myothermal studies the use of blebbistatin, that selectively inhibits myosin activity, has allowed more accurate estimate of the energy expenditure associated with $\mathrm{Ca}^{2+}$ handling in intact cardiac muscle (Pham et al. 2017).

One of the main advantages of skinned preparations is that they allow standardization of the experimental conditions (e.g. composition of the intracellular medium, level of $\mathrm{Ca}^{2+}$-activation), thus minimizing disturbing factors present during the normal twitch of intact cardiac muscle (i.e. variable calcium concentration, hormonal factors, concentrations of metabolic products) (van der Velden et al. 1998; Narolska et al. 2005). The simultaneous measurement of isometric force and ATPase activity in skinned cardiac strips, by means of an enzymatic coupled assay, allows a direct correlation between mechanic and energetic properties of cardiac sarcomeres.

In this method, the re-synthesis of ATP, catalyzed by pyruvate kinase, is enzymatically coupled to the oxidation of nicotinamide dinucleotide (NADH), catalyzed by lactate dehydrogenase. The breakdown of NADH can be determined photometrically by measuring the absorbance of 340nm near-UV light (Glyn and Sleep 1985; Stienen et al. 1993; Kentish and Stienen 1994; Potma et al. 1994; de Tombe and Stienen 1995, 2007). As shown in Fig. 3, simultaneous measurements of maximal isometric tension and ATPase 


\section{Mouse Ventricle}

A
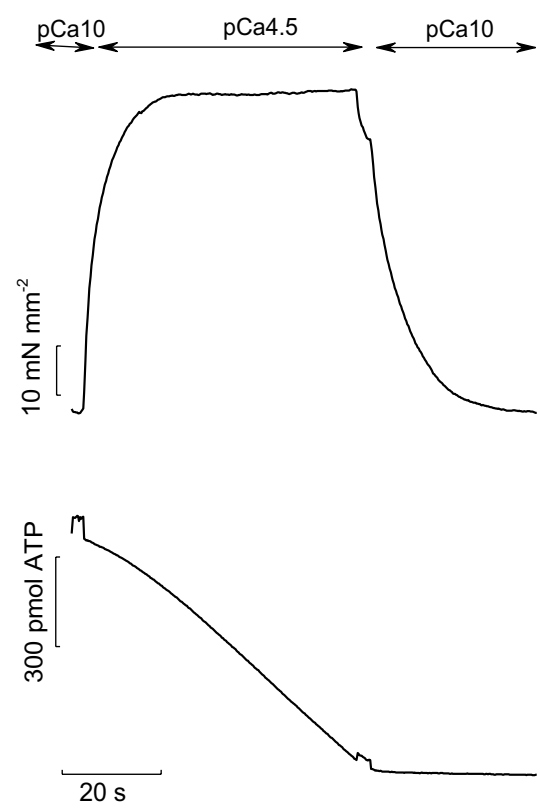

Fig. 3 Simoultaneous measurements of isometric tension and ATPase activity in permeabilized mouse and human ventricular strips. Representative traces of force (top) and ATPase activity (bottom) from permeabilized mouse (A) and human (B) ventricular strips at maximal $\mathrm{Ca}^{2+}$-activation. The muscle strips were activated in saturating $\left[\mathrm{Ca}^{2+}\right]$ solution (pCa 4.5). Isometric force started to develop and NADH absorbance signal started to decline. After force and the slope of the

activity in mouse and human ventricular strips at optimum overlap demonstrate that the slope of the NADH absorbance signal during steady activation is markedly higher in the mouse versus the human preparation. The difference in the amount of maximal tension developed by the two preparations is not enough to account for the higher ATPase found in the mouse myocardium. This indicates that tension cost is much greater in the mouse sarcomeres.

By combining mechanical experiment on single myofibrils (Fig. 1) and simultaneous mechanic and energetic experiments on larger skinned muscle preparations (Fig. 3) one can directly investigate the relation between $\mathrm{CB}$ turnover rate and ATP utilization during isometric muscle contraction. We found that, in disparate types of striated muscles from different species, the slow $k_{\mathrm{REL}}$ measured in myofibrils in our laboratory significantly correlated with the respective energy cost of isometric tension generation measured by different laboratories in skinned preparations from the same muscle type (Fig. 4). In spite of the difference in temperature between the experiments in myofibrils and multicellular skinned preparations and the potential differences in the experimental conditions used in the different laboratories

\section{Human Ventricle}

\section{B}
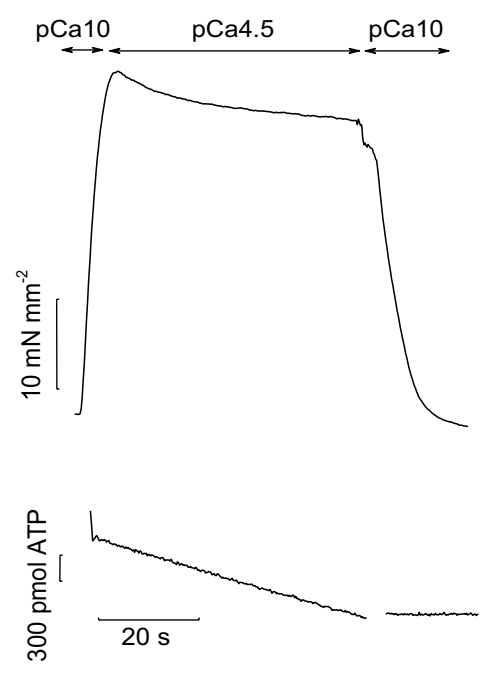

absorbance signal had reached a steady level the muscle strips were relaxed in a low $\left[\mathrm{Ca}^{2+}\right]$ solution (pCa10). The rate of ATP consumption was determined from the slope of the NADH absorbance signal during the last $20 \mathrm{~s}$ of activation. Notably, the slope is much steeper in mouse ventricle strip (A) than in human preparation (B), indicative of a greater ATP consumption to develop force. Sarcomere length $2.2 \mu \mathrm{m}$. Temperature: $20^{\circ} \mathrm{C}$

where the energetic measurements were taken the correlation is rather good. Tension cost data in Fig. 4 are from Narolska et al. (2005) (human atrial and ventricular muscle), from Joumaa et al. (2017) (rabbit psoas), from Ferrantini et al. (2017) (mouse ventricle), and from unpublished data from our laboratory for mouse atrial muscle.

The correlation in Fig. 4 is strong evidence in support of the idea that slow $k_{\mathrm{REL}} \approx g_{\text {app }} \approx$ tension cost. Of note, the positive correlation between these two parameters holds true for muscle types that greatly differ in their actomyosin ATPase rate. In human myocardium, the slow $\beta$ Myosin Heavy Chain $(\beta-\mathrm{MHC})$ isoform is predominantly expressed in the ventricles whereas the fast $\alpha$-MHC isoform is the main molecular motor of the atria. Previous studies on rat and rabbit samples had shown that the fast $\alpha-\mathrm{MHC}$ isoform exhibits a two to three times higher actin-activated ATPase activity (Pope et al. 1980) and actin filament sliding velocity than the slow $\beta$-MHC isoform (Harris et al. 1994). We also had previously found that the much faster relaxation kinetics of atrial vs ventricular myofibrils are consistent with the large difference in CB kinetics between the two MHC isoforms (Piroddi et al. 2007). The proportionality between CB kinetics and tension 


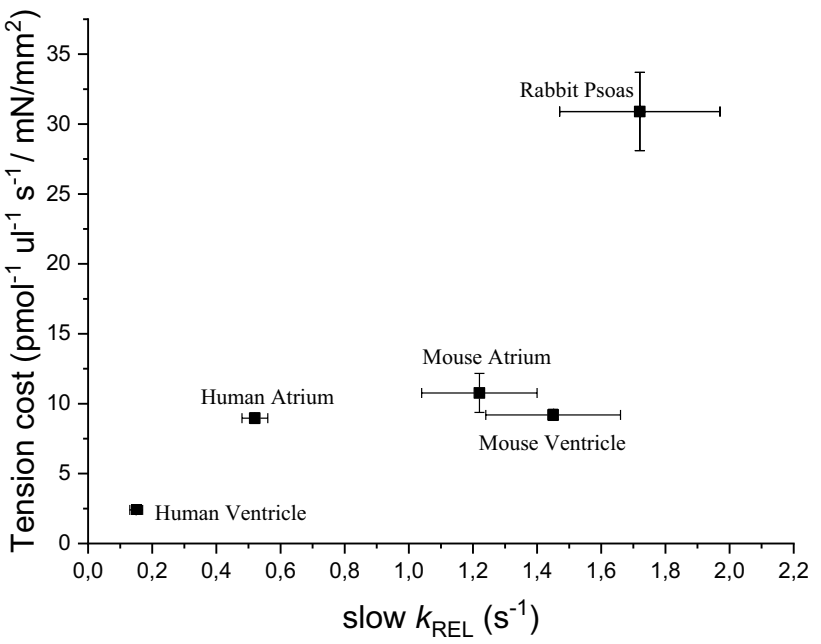

Fig. 4 Relation between slow $k_{\mathrm{REL}}$ and tension cost of different striated muscle preparations. There is a significant correlation $(r=0.90)$ between slow relaxation kinetics of myofibrils $\left(15^{\circ} \mathrm{C}\right)$ and the tension cost of larger permeabilized preparations $\left(20^{\circ} \mathrm{C}\right)$ from a variety of striated muscle tissues in spite of potential differences in the experimental conditions used in the different laboratories were the measurements were taken. Tension cost data of human atrial and ventricular preparations are from Narolska et al. (2005); slow $k_{\mathrm{REL}}$ data for the myofibrils from human atrial and ventricular samples are from Piroddi et al. (2007). Tension cost data of rabbit psoas (consistent with earlier measurements), are from Joumaa et al. (2017); slow $k_{\mathrm{REL}}$ data for the myofibrils from the same muscle are from Kreutziger et al. (2008). Tension cost and slow $k_{\mathrm{REL}}$ data for skinned mouse ventricular trabeculae and myofibrils are from Ferrantini et al. (2017). Tension cost and slow $k_{\mathrm{REL}}$ data for skinned mouse atrial trabeculae and myofibrils are unpublished data from the Authors' laboratory. All data are shown as mean \pm SEM

cost is maintained, since direct measurements of ATPase activity confirm that a fourfold higher slow $k_{\text {REL }}$ (Piroddi et al. 2007) corresponds to a $\sim$ fivefold higher tension cost in atrial vs ventricular human myocardium (Narolska et al. 2005). At variance with human myocardium, in rodents' heart $\alpha-\mathrm{MHC}$ is the main myosin isoform expressed both in the atria and in the ventricles. Of note, as shown in Fig. 4, the kinetics of the slow phase of relaxation and tension cost are rather similar between preparations from the two cardiac chambers of the mouse.

\section{Cardiomyopathy-related alterations of CB kinetics and energetics: increased slow relaxation kinetics underlie higher tension cost}

Myofibril kinetics and energetics have been shown to be altered by several sarcomeric protein mutations associated to genetic cardiomyopathies. In particular, HCM is a genetic disease primarily caused by mutations in the genes encoding sarcomeric proteins (e.g. for reviews see Watkins et al. 2011; Poggesi and Ho 2014). To date, it is unclear how these mutations lead to the cardiac phenotype also because patients carrying the same causal mutation may exhibit different clinical phenotypes.

With the exception of truncation mutations in cardiac myosin binding protein $\mathrm{C}$ for which evidence of haploinsufficiency has been generated (Marston et al. 2009; van Dijk et al. 2009), most HCM mutations result in stable proteins that are incorporated into the sarcomere and may act as poison peptides on the contractile performance, (e.g. Cuda et al. 1993; Bottinelli et al. 1998). In vitro studies on human and animal HCM muscle preparations and isolated proteins suggested that the pathogenic impact of the mutations can be attributed to different mechanisms such as: aberrant $\mathrm{CB}$ dynamics leading to decreased/increased contractility, (e.g. Cuda et al. 1993; Palmiter et al. 2000; Belus et al. 2008; Palmer et al. 2008; Witjas-Paalberends et al. 2014a), increased intrinsic force of the myosin motor (Seebohm et al. 2009; Sommese et al. 2013), increased sarcomeric $\mathrm{Ca}^{2+}$ sensitivity, e.g. (Bottinelli et al. 1998, for a review see Marston 2011). To reconcile the lack of consistent contractility changes in HCM, it has been proposed that HCM sarcomere mutations may lead to increased energy cost of force generation through inefficient or excessive ATPase activity. This results in an energy deficiency of cardiomyocytes that ultimately contributes to the pathogenesis of the disease (Ashrafian et al. 2003). Several studies support this pathogenic hypothesis (Jung et al. 1998; Crilley et al. 2003; Javadpour et al. 2003; Chandra et al. 2005; He et al. 2007; Luedde et al. 2009; Witjas-Paalberends et al. 2014b).

Investigations of the impact of some HCM-related mutations on human cardiac myofibrils revealed a marked acceleration of the apparent rate with which force-generating CBs detach under isometric conditions (Belus et al. 2008; Ferrantini et al. 2009; Witjas-Paalberends et al. 2014a; Ferrantini et al. 2017; Piroddi et al. 2019; Vitale et al. 2019). These studies suggested that a primary mutation-driven effect on myofilaments can be an increase in tension cost. Direct measurement of ATPase activity in multicellular muscle strips from patients confirmed that the energy cost of isometric tension development was higher in HCM patients carrying specific sarcomeric protein mutations compared to controls and to HCM patients without an identified sarcomeric gene mutation ( $\mathrm{HCM}_{\mathrm{smn}}$, sarcomere mutation negative). This was true both for mutations of thick filament proteins, such as the R403Q myosin mutation, and for mutations of thin filament proteins, such as the $\mathrm{K} 280 \mathrm{~N}$ cardiac TnT mutation. While the marked impact of the R403Q mutation in myosin on CB kinetics and sarcomere energetics may be not surprising, the mechanisms of the effects of the K280N mutation in cTnT are much less clear. Pioneer studies, reviewed by Gordon et al. (2000), reported that HCM-associated cTnT mutations may cause changes in regulated acto-S1 ATPase and unloaded thin filament sliding speed. This implies that cTnT can modulate the CB kinetics of the strongly bound 
state in addition to its ability to control the attachment of $\mathrm{CBs}$ to the thin filament.

As shown in Fig. 5A, in all human HCM patient samples analyzed so far (three R403Q beta-MHC, one K280N $\mathrm{cTnT}$, a few sarcomere mutation negative patients) it can be observed that slow $k_{\mathrm{REL}}$ varies in proportion with the respective energy cost of tension development of each individual human cardiac sample independently from the protein carrying the mutation or even in the absence of any mutations (Fig. 5A). Data in Fig. 5A are replotted from published data (Witjas-Paalberends et al. 2014a; Piroddi et al. 2019).

An altered economy of muscle contraction associated to a faster rate of isometric relaxation in myofibrils was also found in an HCM mouse model carrying a mutation (E163R) of cTnT (Ferrantini et al. 2017). In the same study it was also shown that a second mouse model carrying a different mutation in the same protein (R92Q cTnT) did not exhibit the biophysical phenotype described above. As shown in Fig. 5B, a clear correlation between slow $k_{\mathrm{REL}}$ and tension cost is maintained for all human and mouse models investigated independently from species, mutations, and disease states. The slope of the linear relation is relatively high and essentially the same as found in Fig. 5A. The same linear relation is maintained for changes in tension cost and slow $k_{\mathrm{REL}}$ of one order of magnitude. Mouse data in Fig. 5B are

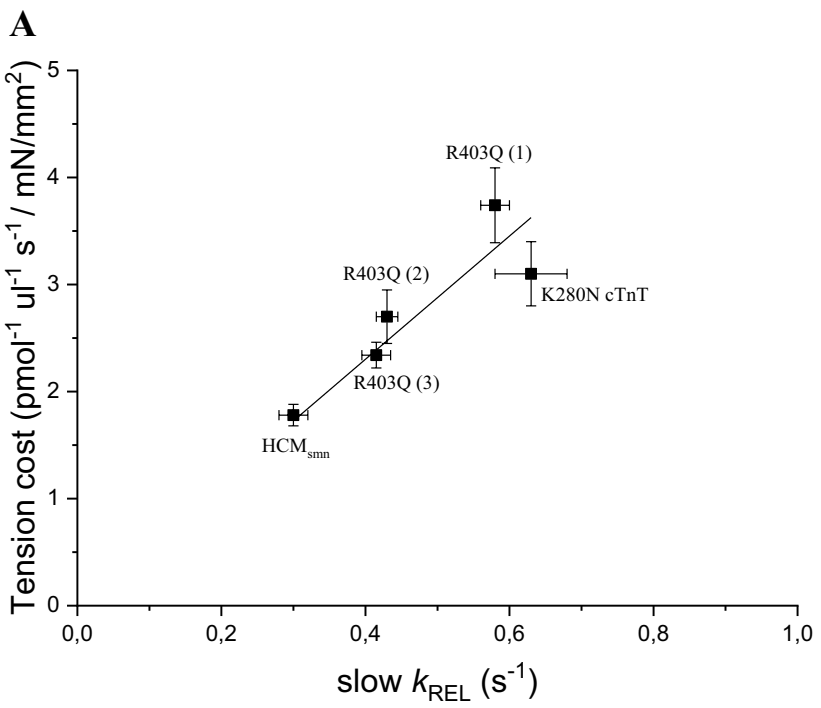

Fig. 5 Relation between slow $k_{\mathrm{REL}}$ and tension cost in ventricular samples from human and mouse HCM models. All data are shown as mean \pm SEM. A Data from human ventricular samples from different HCM patients. The correlation between slow relaxation kinetics of human ventricular myofibrils $\left(15^{\circ} \mathrm{C}\right)$ and tension cost of human myocardial strips $\left(20^{\circ} \mathrm{C}\right)$ can be described by a line with slope 5.75 $(\mathrm{r}=0.99)$. Data from three patients carrying the R403Q mutation in $\beta$-myosin heavy chain [indicated as R403Q (1), (2), and (3)] and from HCM patients without an identified sarcomeric gene mutation (sarcomere mutation negative indicated as $\mathrm{HCM}_{\mathrm{smn}}$ ) are from WitjasPaalberends et al. 2014a. Data from the patient carrying a homozy- from Ferrantini et al. (2017); human data are the same as in Fig. 5A with the individual data of the 3 R403Q patients replaced by the average data from all 3 patients.

\section{Conclusions and implications}

Overall, the data reviewed here provide evidence that a clear linear proportionality exists between the isometric apparent detachment rate of CBs (slow $k_{\mathrm{REL}}$ ) and the energetic cost of tension generation of the sarcomere measured in preparations from the same muscle sample. The relationship holds true among a variety of striated muscles from different species and also when CB kinetics is altered by sarcomeric mutations, strengthening the expectation of the simple two-state CB model (Brenner 1988) that the kinetics of CB detachment under isometric conditions along forward transitions ( $\mathrm{g} \sim$ slow $k_{\mathrm{REL}}$ ) correlates linearly with the amount of ATP spent per unit force.

The experimental observations that sarcomeric protein mutations associated to $\mathrm{HCM}$ are often associated to increased tension cost (Fig. 5) may, in principle, suffer from artifacts due to the structural remodeling often undergone by cardiac muscle in HCM. In fact, cardiomyocyte disarray may artificially decrease isometric tension and increase the

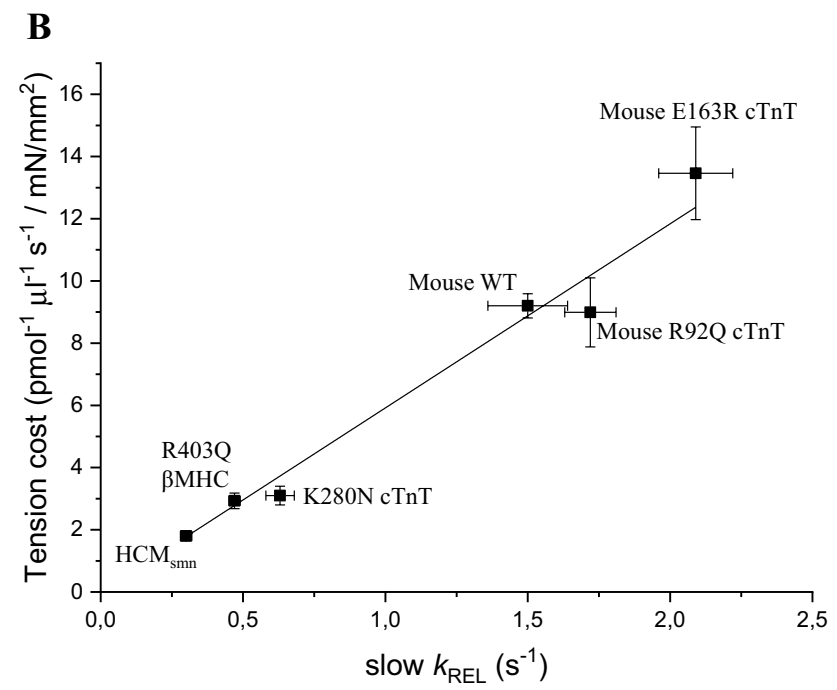

gous mutation in cTnT (K280N) are from Piroddi et al. 2019. B Data from human and mouse models. The correlation between slow relaxation kinetics of ventricular myofibrils $\left(15{ }^{\circ} \mathrm{C}\right)$ and tension cost of myocardial strips $\left(20^{\circ} \mathrm{C}\right)$ can be described by a line with slope 5.92 $(\mathrm{r}=0.99)$. Human data are the same as in panel A with the individual data of the 3 R403Q patients replaced by the average data from all 3 patients. Mouse data are taken from Ferrantini et al. (2017). Independently from species, mutations, and disesase state, slow $k_{\mathrm{REL}}$ measured in myofibrils correlates linearly with the tension cost measured in multicellular muscle strips from the same samples 
"isometric" ATPase of multicellular preparations resulting in artificial increase in the tension cost. To address the problem we have developed techniques to reconstruct the 3D structure of the whole ventricular strips used for mechanical and energetic experiments (Vitale et al. 2019). Following 3D image reconstruction of the strips at sub-micrometer spatial resolution, cardiomyocyte orientation across and along the strips is determined. Statistics of spatial disarray are derived and correlated to mechanical and energetic data. Preliminary results (Vitale et al. 2019) do not highlight structural differences between donor and HCM strips strengthening the conclusion that HCM mutations primarily alter apparent CB kinetics and impair sarcomere energetics.

Is the increase in the energy cost of isometric tension generation, observed with a number of HCM-associated mutations, expected to alter the overall cost of the actual cardiac cycle? Though our data do not say much about the energetic cost of contraction under mechanical conditions that are not isometric, the specific impact of a number of mutations associated to HCM on isometric CB kinetics and isometric tension cost may help explaining some yet unresolved clinical features of HCM. Generation of large amounts of isometric tension in the heart only occurs in the walls of the left ventricle (LV), especially during isovolumic contraction, though high tension is also maintained during LV ejection. Though the mutant proteins may be expressed in all cardiac chambers, the disease specifically affects the LV causing an asymmetric hypertrophy most often affecting the interventricular septum. Theoretical models challenge the assumption of uniform myocardial wall tension in the LV contraction (DeAnda et al. 1998) and indicate the septum as the region where the highest stress is generated. This may be also supported by studies that show greater energy demands in the septum (Dunn 1984). Superimposing regional mechanical and metabolic differences on HCM myocytes might lead to localized uncompensated energetic load and hence asymmetric hypertrophy.

Does the increased energy cost of tension generation and the consequent energy impairment of HCM cardiomyocytes contribute to the asymmetric LV hypertrophy observed in HCM? Using magnetic resonance spectroscopy, a reduction in the cardiac PCr to ATP ratio, a measure of energetic status, has been reported in HCM patients with LV hypertrophy (Crilley et al. 2003). In the same study, abnormal mean PCr/ ATP ratios have also been found in pre-hypertrophic carriers of HCM mutations (genotype positive/clinical phenotype negative subjects) in which the energetic alterations cannot be attributed to the confounding hypertrophy. Therefore, it can be proposed that a primary stimulus for hypertrophy may be the energetic defect itself. If energy generation cannot match demand, cytosolic $\mathrm{Ca}^{2+}$ re-uptake/removal at the end of each contraction is compromised, particularly due to the extreme energy requirements of SERCA2, the sarcoplasmic reticulum $\mathrm{Ca}^{2+}$ re-uptake pump. The resultant prolonged cytosolic $\mathrm{Ca}^{2+}$ transient may be able to trigger downstream hypertrophy pathways.

Acknowledgements This research was supported by the European Union's Horizon 2020 research and innovation programme, project SILICOFCM Grant Agreement No. 777204.

Open Access This article is distributed under the terms of the Creative Commons Attribution 4.0 International License (http://creativeco mmons.org/licenses/by/4.0/), which permits unrestricted use, distribution, and reproduction in any medium, provided you give appropriate credit to the original author(s) and the source, provide a link to the Creative Commons license, and indicate if changes were made.

\section{References}

Alpert NR, Mulieri LA, Hasenfuss G (1991) Myocardial chemomechanical energy transduction. In: Fozzard HA, Jennings RB, Haber E, Katz AM (eds) The heart and cardiovascular system: scientific foundations, 2nd edn. Raven Press Publishers, New York, NY, pp 111-128

Ashrafian H, Redwood C, Blair E, Watkins H (2003) Hypertrophic cardiomyopathy: a paradigm for myocardial energy depletion. Trends Genet 19:263-268

Belus A, Piroddi N, Scellini B, Tesi C, D’Amati G, Girolami F, Yacoub M, Cecchi F, Olivotto I, Poggesi C (2008) The familial hypertrophic cardiomyopathy-associated myosin mutation R403Q accelerates tension generation and relaxation of human cardiac myofibrils. J Physiol 586:3639-3644

Belus A, Piroddi N, Ferrantini C, Tesi C, Cazorla O, Toniolo L, Drost M, Mearini G, Carrier L, Rossi A, Mugelli A, Cerbai E, van der Velden J, Poggesi C (2010) Effects of chronic atrial fibrillation on active and passive force generation in human atrial myofibrils. Circ Res 107:144-152

Bers DM (2001) Excitation-contraction coupling and cardiac contractile force, 2nd edn. Kluwer Academic Publishers, Doordrecht

Bottinelli R, Coviello DA, Redwood CS, Pellegrino MA, Maron BJ, Spirito P, Watkins H, Reggiani C (1998) A mutant tropomyosin that causes hypertrophic cardiomyopathy is expressed in vivo and associated with an increased calcium sensitivity. Circ Res 82:106-115

Brenner B (1988) Effect of $\mathrm{Ca}^{2+}$ on cross-bridge turnover kinetics in skinned single rabbit psoas fibers: implications for regulation of muscle contraction. Proc Natl Acad Sci USA 85:3265-3269

Caputo C, Edman KA, Lou F, Sun YB (1994) Variation in myoplasmic $\mathrm{Ca}^{2+}$ concentration during contraction and relaxation studied by the indicator fluo-3 in frog muscle fibres. J Physiol 478:137-148

Chandra M, Tschirgi ML, Tardiff JC (2005) Increase in tensiondependent ATP consumption induced by cardiac troponin T mutation. Am J Physiol Heart Circ Physiol 289:H2112-H2119

Cleworth DR, Edman KA (1972) Changes in sarcomere length during isometric tension development in frog skeletal muscle. J Physiol 227:1-17

Crilley JG, Boehm EA, Blair E, Rajagopalan B, Blamire AM, Styles P, McKenna WJ, Ostman-Smith I, Clarke K, Watkins H (2003) Hypertrophic cardiomyopathy due to sarcomeric gene mutations is characterized by impaired energy metabolism irrespective of the degree of hypertrophy. J Am Coll Cardiol 41:1776-1782 
Cuda G, Fananapazir L, Zhu WS, Sellers JR, Epstein ND (1993) Skeletal muscle expression and abnormal function of beta-myosin in hypertrophic cardiomyopathy. J Clin Investig 91:2861-2865

de Tombe PP, Stienen GJ (1995) Protein kinase A does not alter economy of force maintenance in skinned rat cardiac trabeculae. Circ Res 76:734-741

de Tombe PP, Stienen GJ (2007) Impact of temperature on cross-bridge cycling kinetics in rat myocardium. J Physiol 584:591-600

DeAnda A Jr, Komeda M, Moon MR, Green GR, Bolger AF, Nikolic SD, Daughters GT 2nd, Miller DC (1998) Estimation of regional left ventricular wall stresses in intact canine hearts. Am J Physiol 275:H1879-H1885

Dunn RB (1984) High energy phosphate depletion and lactate accumulation in the interventricular septum and left ventricular free wall of the dog after total coronary occlusion. Circ Res 54:405-413

Edman KA, Flitney FW (1982) Laser diffraction studies of sarcomere dynamics during 'isometric' relaxation in isolated muscle fibres of the frog. J Physiol 329:1-20

Ferrantini C, Belus A, Piroddi N, Scellini B, Tesi C, Poggesi C (2009) Mechanical and energetic consequences of HCM-causing mutations. J Cardiovasc Transl Res 2:441-451

Ferrantini C, Coppini R, Pioner JM, Gentile F, Tosi B, Mazzoni L, Scellini B, Piroddi N, Laurino A, Santini L, Spinelli V, Sacconi L, De Tombe P, Moore R, Tardiff J, Mugelli A, Olivotto I, Cerbai E, Tesi C, Poggesi C (2017) Pathogenesis of hypertrophic cardiomyopathy is mutation rather than disease specific: a comparison of the cardiac troponin T E163R and R92Q mouse models. J Am Heart Assoc 6:e05407

Gao WD, Perez NG, Marban E (1998) Calcium cycling and contractile activation in intact mouse cardiac muscle. J Physiol 507:175-184

Gibbs CL, Loiselle DS, Wendt IR (1988) Activation heat in rabbit cardiac muscle. J Physiol 395:115-130

Glyn H, Sleep J (1985) Dependence of adenosine triphosphate activity of rabbit psoas muscle fibres and myofibrils on substrate concentrations. J Physiol 365:259-276

Gordon AM, Homsher E, Regnier M (2000) Regulation of contraction in striated muscle. Physiol Rev 80:853-924

Harris DE, Work SS, Wright RK, Alpert NR, Warshaw DM (1994) Smooth, cardiac and skeletal muscle myosin force and motion generation assessed by cross-bridge mechanical interactions in vitro. J Muscle Res Cell Motil 15:11-19

He H, Javadpour MM, Latif F, Tardiff JC, Ingwall JS (2007) R-92L and R-92W mutations in cardiac troponin T lead to distinct energetic phenotypes in intact mouse hearts. Biophys J 93:1834-1844

Holubarsch CH, Hasenfuss G, Just H, Blanchard EM, Mulieri LA, Alpert NR (1991) Modulation of myothermal economy of isometric force generation by positive inotropic interventions in the guinea pig myocardium. Cardioscience 1:33-41

Huxley AF, Simmons RM (1970) Rapid 'give' and the tension 'shoulder' in the relaxation of frog muscle fibres. J Physiol 210:32P-33P

Huxley AF, Simmons RM (1973) Mechanical transients and the origin of muscular force. Cold Spring Harb Symp Quant Biol 37:669-680

Javadpour MM, Tardiff JC, Pinz I, Ingwall JS (2003) Decreased energetics in murine hearts bearing the R92Q mutation in cardiac troponin T. J Clin Investig 112:768-775

Jeong MY, Lin YH, Wennersten SA, Demos-Davies KM, Cavasin MA, Mahaffey JH, Monzani V, Saripalli C, Mascagni P, Reece TB, Ambardekar AV, Granzier HL, Dinarello CA, McKinsey TA (2018) Histone deacetylase activity governs diastolic dysfunction through a nongenomic mechanism. Sci Transl Med 10(427):eaao0144

Joumaa V, Fitzowich A, Herzog W (2017) Energy cost of isometric force production after active shortening in skinned muscle fibres. J Exp Biol 220:1509-1515
Jung WI, Sieverding L, Breuer J, Hoess T, Widmaier S, Schmidt O, Bunse M, van Erckelens F, Apitz J, Lutz O, Dietze GJ (1998) 31P NMR spectroscopy detects metabolic abnormalities in asymptomatic patients with hypertrophic cardiomyopathy. Circulation 97:2536-2542

Kentish JC, Stienen GJM (1994) Differential effects of length on maximum force production and myofibrillar ATPase activity in rat skinned cardiac muscle. J Physiol 475:175-184

Kreutziger KL, Piroddi N, Scellini B, Tesi C, Poggesi C, Regnier M (2008) Thin filament $\mathrm{Ca}^{2+}$ binding properties and regulatory unit interactions alter kinetics of tension development and relaxation in rabbit skeletal muscle. J Physiol 586:3683-3700

Kreutziger KL, Piroddi N, McMichael JT, Tesi C, Poggesi C, Regnier M (2011) Calcium binding kinetics of troponin C strongly modulate cooperative activation and tension kinetics in cardiac muscle. Mol Cell Cardiol 50:165-174

Luedde M, Flogel U, Knorr M, Grundt C, Hippe HJ, Brors B, Frank D, Haselmann U, Antony C, Voelkers M, Schrader J, Most P, Lemmer B, Katus HA, Frey N (2009) Decreased contractility due to energy deprivation in a transgenic rat model of hypertrophic cardiomyopathy. J Mol Med 87:411-422

Luo Y, Davis JP, Smillie LB, Rall JA (2002) Determinants of relaxation rate in rabbit skinned skeletal muscle fibres. J Physiol (Lond) 545:887-901

Maron BJ, Ommen SR, Semsarian C, Spirito P, Olivotto I, Maron MS (2014) Hypertrophic cardiomyopathy: present and future, with translation into contemporary cardiovascular medicine. J Am Coll Cardiol 64:83-99

Marston SB (2011) How do mutations in contractile proteins cause the primary familial cardiomyopathies? J Cardiovasc Transl Res 4:245-255

Marston S, Copeland O, Jacques A, Livesey K, Tsang V, McKenna WJ, Jalilzadeh S, Carballo S, Redwood C, Watkins H (2009) Evidence from human myectomy samples that MYBPC3 mutations cause hypertrophic cardiomyopathy through haploinsufficiency. Circ Res 105:219-222

Narolska NA, van Loon RB, Boontje NM, Zaremba R, Penas SE, Russell J, Spiegelenberg SR, Huybregts MA, Visser FC, de Jong JW et al (2005) Myocardial contraction is 5-fold more economical in ventricular than in atrial human tissue. Cardiovasc Res 65:221-229

Neagoe C, Kulke M, del Monte F, Gwathmey JK, de Tombe PP, Hajjar RJ, Linke WA (2002) Titin isoform switch in ischemic human heart disease. Circulation 106:1333-1341

Palmer S, Kentish JC (1998) Roles of $\mathrm{Ca}^{2+}$ and crossbridge kinetics in determining the maximum rates of $\mathrm{Ca}^{2+}$ activation and relaxation in rat and guinea pig skinned trabeculae. Circ Res 83:179-186

Palmer BM, Wang Y, Teekakirikul P, Hinson JT, Fatkin D, Strouse S, Vanburen P, Seidman CE, Seidman JG, Maughan DW (2008) Myofilament mechanical performance is enhanced by R403Q myosin in mouse myocardium independent of sex. Am J Physiol Heart Circ Physiol 294:H1939-H1947

Palmiter KA, Tyska MJ, Haeberle JR, Alpert NR, Fananapazir L, Warshaw DM (2000) R403Q and L908V mutant beta-cardiac myosin from patients with familial hypertrophic cardiomyopathy exhibit enhanced mechanical performance at the single molecule level. J Muscle Res Cell Motil 21:609-620

Pham T, Tran K, Mellor KM, Hickey A, Power A, Ward ML, Taberner A, Han JC, Loiselle D (2017) Does the intercept of the heat-stress relation provide an accurate estimate of cardiac activation heat? $\mathrm{J}$ Physiol 595:4725-4733

Piroddi N, Tesi C, Pellegrino MA, Tobacman LS, Homsher E, Poggesi C (2003) Contractile effects of the exchange of cardiac troponin for fast skeletal troponin in rabbit psoas single myofibrils. J Physiol 552:17-31 
Piroddi N, Belus A, Scellini B, Tesi C, Giunti G, Cerbai E, Mugelli A, Poggesi C (2007) Tension generation and relaxation in single myofibrils from human atrial and ventricular myocardium. Pflugers Arch 454:63-73

Piroddi N, Witjas-Paalberends ER, Ferrara C, Ferrantini C, Vitale G, Scellini B, Wijnker PJM, Sequiera V, Dooijes D, Dos Remedios C, Schlossarek S, Leung MC, Messer A, Ward DG, Biggeri A, Tesi C, Carrier L, Redwood CS, Marston SB, van der Velden J, Poggesi C (2019) The homozygous K280N troponin T mutation alters cross-bridge kinetics and energetics in human HCM. J Gen Physiol 151:18-29

Poggesi C, Ho CY (2014) Muscle dysfunction in hypertrophic cardiomyopathy: what is needed to move to translation? J Muscle Res Cell Motil 35:37-45

Poggesi C, Tesi C, Stehle R (2005) Sarcomeric determinants of striated muscle relaxation kinetics. Pflugers Arch 449:505-517

Pope B, Hoh JFY, Weeds A (1980) The ATPase activities of rat cardiac myosin isozymes. FEBS Lett 118:205-208

Potma EJ, Stienen GJM, Barends JPF, Elzinga G (1994) Myofibrillar ATPase activity and mechanical performance of skinned fibres from rabbit psoas muscle. J Physiol 474:303-317

Scellini B, Piroddi N, Poggesi C, Tesi C (2010) Extraction and replacement of the tropomyosin-troponin complex in isolated myofibrils. Adv Exp Med Biol 682:163-174

Seebohm B, Matinmehr F, Kohler J, Francino A, Navarro-Lopez F, Perrot A, Ozcelik C, McKenna WJ, Brenner B, Kraft T (2009) Cardiomyopathy mutations reveal variable region of myosin converter as major element of cross-bridge compliance. Biophys $\mathrm{J}$ 97:806-824

Siththanandan VB, Tobacman LS, Van Gorder N, Homsher E (2009) Mechanical and kinetic effects of shortened tropomyosin reconstituted into myofibrils. Pflugers Arch 458:761-776

Solzin J, Iorga B, Sierakowski E, Gomez Alcazar DP, Ruess DF, Kubacki T, Zittrich S, Blaudeck N, Pfitzer G, Stehle R (2007) Kinetic mechanism of the $\mathrm{Ca}^{2+}$-dependent switch-on and switch off of cardiac troponin in myofibrils. Biophys J 93:3917-3931

Sommese RF, Sung J, Nag S, Sutton S, Deacon JC, Choe E, Leinwand LA, Ruppel K, Spudich JA (2013) Molecular consequences of the R453C hypertrophic cardiomyopathy mutation on human beta-cardiac myosin motor function. Proc Natl Acad Sci USA 110:12607-12612

Stehle R, Tesi C (2017) Kinetic coupling of phosphate release, force generation and rate-limiting steps in the cross-bridge cycle. J Muscle Res Cell Motil 38:275-289

Stehle R, Krüger M, Pfitzer G (2002) Force kinetics and individual sarcomere dynamics in cardiac myofibrils after rapid $\mathrm{Ca}^{2+}$ changes. Biophys J 83:2152-2161

Stehle R, Solzin J, Iorga B, Poggesi C (2009) Insights into the kinetics of $\mathrm{Ca}^{2+}$-regulated contraction and relaxation from myofibril studies. Pflugers Arch 458:337-357

Stienen GJM, Papp Z, Elzinga G (1993) Calcium modulates the influence of length changes on the myofibrillar adenosine triphosphatase activity in rat skinned cardiac trabeculae. Pflugers Arch 425:199-207
Tesi C, Piroddi N, Colomo F, Poggesi C (2002) Relaxation kinetics following sudden $\mathrm{Ca}^{2+}$ reduction in single myofibrils from skeletal muscle. Biophys J 83:2142-2151

van der Velden J, Moorman AF, Stienen GJM (1998) Age-dependent changes in myosin composition correlate with enhanced economy of contraction in guinea-pig hearts. J Physiol 507:497-510

van Dijk SJ, Dooijes D, dos Remedios C, Michels M, Lamers JM, Winegrad S, Schlossarek S, Carrier L, ten Cate FJ, Stienen GJ, van der Velden J (2009) Cardiac myosin-binding protein C mutations and hypertrophic cardiomyopathy: haploinsufficiency, deranged phosphorylation, and cardiomyocyte dysfunction. Circulation 119:1473-1483

Vikhorev PG, Smoktunowicz N, Munster AB, Copeland O, Kostin S, Montgiraud C, Messer AE, Toliat MR, Li A, Dos Remedios CG, Lal S, Blair CA, Campbell KS, Guglin M, Richter M, Knöll R, Marston SB (2017) Abnormal contractility in human heart myofibrils from patients with dilated cardiomyopathy due to mutations in TTN and contractile protein genes. Sci Rep. 7:14829

Vitale G, Lazzeri E, Costantini I, Giardini F, Mazzamuto G, Crocini C, Piroddi N, Scellini B, Pioner MJ, Ferrantini C, Tesi C, Pavone FS, Sacconi L, Poggesi C (2019) Advanced morpho-functional analysis on ventricular and atrial tissue reveals cross-bridge kinetics alterations and sarcomere energetic impairment in hcm patients. Biophys J 116(Supplement 1):29a

Wahr PA, Johnson JD, Rall JA (1998) Determinants of relaxation rate in skinned frog skeletal muscle fibres. Am J Physiol 274:C1608-C1615

Walker JS, Walker LA, Margulies K, Buttrick P, de Tombe P (2011) Protein kinase A changes calcium sensitivity but not crossbridge kinetics in human cardiac myofibrils. Am J Physiol Heart Circ Physiol 301:H138-H146

Watkins H, Ashrafian H, Redwood C (2011) Inherited cardiomyopathies. N Engl J Med 364:1643-1656

Witjas-Paalberends ER, Ferrara C, Scellini B, Piroddi N, Montag J, Tesi C, Stienen GJ, Michels M, Ho CY, Kraft T, Poggesi C, van der Velden J (2014a) Faster cross-bridge detachment and increased tension cost in human hypertrophic cardiomyopathy with the R403Q MYH7 mutation. J Physiol 592:3257-3272

Witjas-Paalberends ER, Guclu A, Germans T, Knaapen P, Harms HJ, Vermeer AM, Christiaans I, Wilde AA, Remedios CD, Lammertsma AA, van Rossum AC, Stienen GJ, van Slegtenhorst M, Schinkel AF, Michels M, Ho CY, Poggesi C, van der Velden J (2014b) Gene-specific increase in energetic cost of contraction in hypertrophic cardiomyopathy caused by thick filament mutations. Cardiovasc Res 103:248-257

Publisher's Note Springer Nature remains neutral with regard to jurisdictional claims in published maps and institutional affiliations. 